Case Report

\title{
GIANT COLONIC POLYPS IN PEDIATRIC PATIENT WITH FAMILIAL ADENOMATOUS POLYPOSIS
}

\author{
Zlatko Djurić $^{1}$, Aleksandar Nagorni ${ }^{2}$, Maja Jovičić-Milentijević ${ }^{3}$, Jelisaveta Maksimović ${ }^{4}$ \\ ${ }^{1}$ Pediatric Clinic, Department of Gastroenterology, Clinical Center of Niš, University of Niš, Faculty of Medicine, Niš, \\ Serbia \\ ${ }^{2}$ Clinic of Gastroenterology and Hepatology, Clinical Center of Niš, University of Niš, Faculty of Medicine, Niš, Serbia \\ ${ }^{3}$ Institute of Pathology, Clinical Center of Niš, University of Niš, Faculty of Medicine, Niš, Serbia \\ ${ }^{4}$ University of Niš, Faculty of Medicine, Niš, Serbia
}

\begin{abstract}
This article describes a 16-year-old girl with signs of malnutrition, rectal bleeding, hypoalbuminemia and anemia. At the age of 6 months she was operated on for hepatoblastoma. Last two years she has been followed by endocrinologist because of amenorrhea. Her family history was unremarkable. Esophagogastroduodenoscopy revealed multiple small gastric polyps. Colonoscopy showed a few polyps in rectum and hundreds of them (3-25 mm in diameter) in sigmoid and descending colon. Giant colonic polyps at splenic flexure region prevented further passage of the scope. The diagnosis of FAP was established based on the endoscopy and pathology of colonic polyps removed by snare polypectomy. Extraintestinal manifestations of FAP have not been detected. The patient underwent prophylactic surgery (restorative proctocolectomy). The post-operative course was uneventful. In our knowledge, this is the first report of giant colonic polyps in a pediatric patient suffering from FAP. This clinical syndrome can be presented not only with different genetic backgrounds and diverse clinical pictures but also with intriguing endoscopic findings.
\end{abstract}

Key words: polyps, familial, polyposis, pediatric, patient.

\section{Introduction}

Familial adenomatous polyposis (FAP) is the most frequent inherited polyposis syndrome caused by mutation in the adenomatous polyposis gene (APC) on chromosome $5 q 21$ [1] The estimated prevalence of FAP is between 1:5000 and 1:17000 [2]. This syndrome is inherited in an autosomal dominant manner, although around 20-30\% patients have de novo mutations [1]. It is characterized by the early onset (second or third decade of life) of hundreds to thousands colorectal adenomas. Without prophylactic colectomy, all affected patients develop colorectal cancer (CRC) by the age of 40-50 years. In the remainder of gastrointestinal tract adenomas can be found in stomach and small bowel. The most common extraintestinal manifestations of FAP are: congenital hypertrophy of the retinal pigment epithelium (CHRPE), desmoid tumors, epidermoid cysts and osteomas [3]. In addition, FAP is associated with an increased risk of malignant tumors at other sites including: thyroid (in $2-7 \%$ cases), liver $(<2 \%$ cases) and brain $(<1 \%$ cases) [4]. Patients with milder forms of this syndrome - attenuated FAP and MYTUH

Correspondence to: Zlatko Djurić, Ph.D.

Pediatric Clinic, Department of Gastroenterology, Clinical Center of Niš University of Niš, Faculty of Medicine, Niš, Serbia

48 Dr. Zoran Djindjić Blvd., Niš 18000, Serbia

Phone: +381641137270

E-mail: zldjuric@yahoo.com

Received July $20^{\text {th }}, 2017$, Accepted September $21^{\text {st }}, 2017$ associated polyposis rarely have intestinal manifestations in childhood.

\section{Case Report}

A 16-year-old girl presented with rectal bleeding and signs of malnutrition (body height $155 \mathrm{~cm}$ (P12), body weight $29 \mathrm{~kg}$, BMI 12,1 kg/m²). At the age of 6 months she was operated on for hepatoblastoma. At the age of 11 months 3 cysts in her left kidney were revealed by ultrasound. Last two years she has been followed by endocrinologist because of amenorrhea. Her family history was unremarkable. The initial work up showed anemia (Hb 9.8X10, Er 4.68X10, Hct 32.9\%) and hypoalbuminemia (S-Alb $24.7 \mathrm{~g} / \mathrm{L}$ ). Stool was negative on ova and parasites, bacteria and Clostridium difficile toxins A/B. Esophagogastroduodenoscopy revealed multiple small gastric polyps. Colonoscopy showed a few polyps in rectum and hundreds of them (3-25 mm in diameter) in sigmoid and descending colon. Giant colonic polyps (Fig.1) at splenic flexure region prevented further passage of the scope. Pathology of polyps removed by snare polypectomy showed tubulovillous adenomas with low grade dysplasia (Fig. 2). On fundoscopic exam signs of CHRPE have not been seen. Abdominal ultrasound and magnetic resonance imaging excluded presence of desmoid tumors, but revealed three small cysts in the left kidney.

The patient underwent restorative proctocolectomy at another hospital. Post-operative pathology has not proved CRC. 


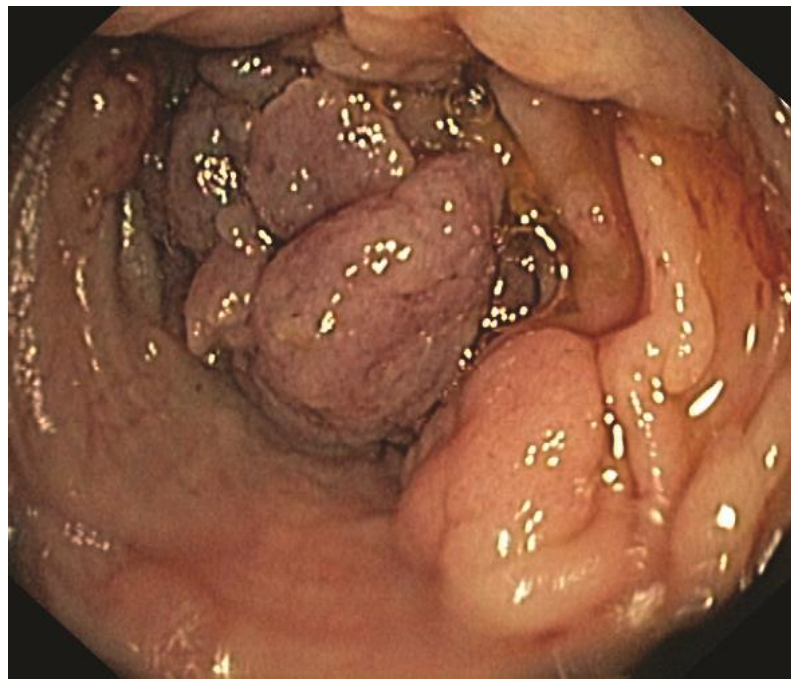

Fig. 1 Large colonic polyps at splenic flexure region

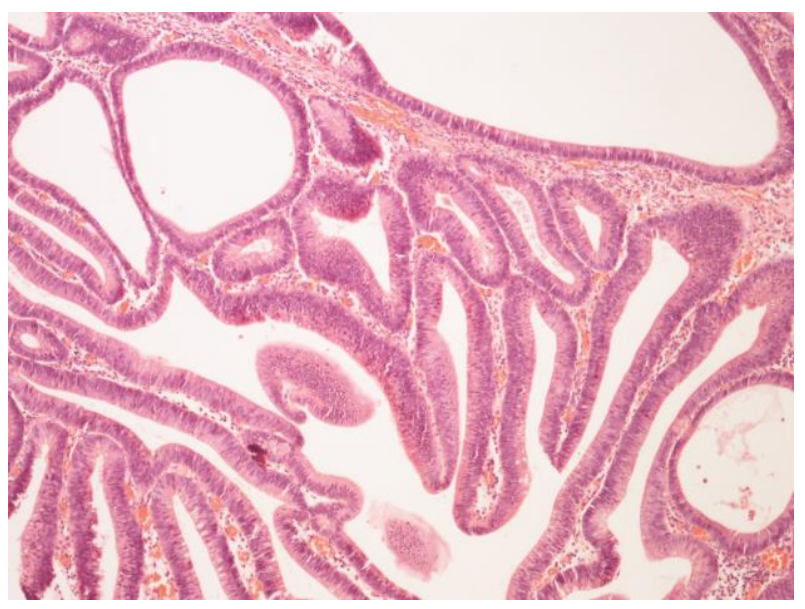

Fig. 2 Tubulovillous adenoma with low grade dysplasia

\section{Discussion}

Herein we describe a case of an adolescent female, with a unique endoscopic finding - giant colonic polyps at the splenic flexure region. The diagnosis of FAP was established based on the endoscopy and pathology of colonic polyps, removed by snare polypectomy. Unfortunately, the genetic test for FAP was not available in our institution. Therefore, we were not able to determine the type of mutation associated with this unusual endoscopic finding and the severe course of the disease.

Up until now more than 800 mutations of APC gene have been discovered in patients with classic and attenuated forms of FAP. Genotype-phenotype correlation studies indicated that mutation between codons 1250 and 1464 of APC gene and especially mutation at codon
1309 were associated with a severe clinical phenotype in FAP patients [5]. The genetic screening should be performed in all first degree relatives of FAP patients and should be started at the age of 10 years [4]. Families need to be informed that mutation can be found in only $70-90 \%$ cases.

Biannual flexible colonoscopy of at-risk children generally should begin at the age 10 years [4].

If the FAP symptoms become apparent at an earlier age or there is positive family history of: aggressive disease or mutation associated with a severe clinical phenotype, earlier colonoscopy is reasonable. Upper endoscopic surveillance (with end- and side-viewing scopes) should begin at the time when colonic adenomas are identified, or at the age of 20-25 years.

Rectal bleeding is the most common clinical sign described in patients with FAP. All clinical signs and laboratory findings in our patient, including rectal bleeding, severe malnutrition, amenorrhea, anemia, and hypoalbuminemia, were caused by the severe course of the disease. Our patient' history indicated that she was operated on in early infancy for hepatoblastoma. The risk of hepatoblastoma is 850 times greater in patients with FAP than in the general population; in most cases, it occurs within the first three years of life [6]. There is still no consensus regarding hepatoblastoma screening in patients with FAP. Some clinicians recommend that liver palpation, abdominal ultrasound, and alpha-fetoprotein level in serum should be done every 3-6 months during the first 5 years of life [7].

The patient underwent restorative proctocolectomy due to the presence of significant symptoms which is one of absolute indications for prophylactic surgery in patients with FAP according to recently published American College of Gastroenterology clinical guideline [8].

Restorative proctocolectomy is the surgical procedure of choice in the vast majority of FAP patients [9]. Alternatively, ileorectal anastomosis can be done in patients with a small number of adenomas in rectum. Regardless of the type of surgical procedure, regular endoscopic surveillance is a must for all operated patients in order to detect polyp recurrence.

\section{Conclusion}

In our knowledge, this is the first report of giant colonic polyps in a pediatric patient suffering from FAP. This is the most common inherited polyposis syndrome in childhood that can be presented not only with different genetic backgrounds and diverse clinical pictures, but also with intriguing endoscopic findings. 


\section{References}

1. Barnard J. Screening and surveillance recommendations for pediatric gastrointestinal polyposis syndromes. J Pediatr Gastroentrol Nutr 2009; 48:S75-S78.

2. Corredor J, Wambach J, Barnard J. Gastrointestinal polyps in children: advances in molecular genetics, diagnosis and management. J Pediatr 2001; 138:621-8.

3. Jung I, Gurzu S, Turdean GS. Current status of gastrointestinal polyposis syndromes. World J Gastrointest Oncol 2015; 7:347-55.

4. Septer S, Lawson CE, Anant S, Attard T. Famiial adenomatous polyposis in pediatrics: natural history, emerging surveillance and management protocols, chemopreventive strategies, and areas of ongoig debate. Fam Cancer 2016; 15:477-85.

5. Hyer W. Pediatric polyposis syndromes. In: Wyllie R. Hyams J, Marsha K. Pediaric Gastrointestinal and liver disease. 5th ed, Elsevier, Inc: Philadelphia, 2016, pp 496-507.
6. Giardiello FM, Peterson GM, Brensinger JD, et al. Hepatoblastoma and APC gene mutation in familial adenomatous polyposis. Gut 1996; 39:867-9.

7. Hirschman BA, Pollock BH, Tomlinson GE. The spectrum of APC mutations in children with hepatoblastoma from familial adenomatous polyposis kindreds. J Pediatr 2005; 147:263-6.

8. Syngal S, Brand RE, Churc JM, Giardiello FM, Hampel HL, Burt RW; American College of Gastroenterology. ACG clinical guideline: Genetic testing and management of hereditary gastrointestinal cancer syndromes. Am J Gastroenterol 2015; 110: 223-62

9. Kennedy RD, Zarroug AE, Moir CR. Ileal pouch anal anastomosis in pediatric familial adenomatous polyposis. A 24-year review of operative technique and patient outcomes. J Pediatr Surg 2014; 49: 1409-12. 